\title{
Phase-Field Model of Mode III Dynamic Fracture
}

\author{
Alain Karma ${ }^{1}$, David A. Kessler ${ }^{2}$, and Herbert Levine ${ }^{3}$ \\ ${ }^{1}$ Department of Physics, Northeastern University, Boston MA 02215 \\ ${ }^{2}$ Department of Physics, Bar-Ilan University, Ramat-Gan, Israel \\ ${ }^{3}$ Department of Physics, University of California, San Diego, La Jolla, CA 92093-0319
}

(May 28, 2018)

\begin{abstract}
We introduce a phenomenological continuum model for mode III dynamic fracture that is based on the phase-field methodology used extensively to model interfacial pattern formation. We couple a scalar field, which distinguishes between "broken" and "unbroken" states of the system, to the displacement field in a way that consistently includes both macroscopic elasticity and a simple rotationally invariant short scale description of breaking. We report two-dimensional simulations that yield steady-state crack motion in a strip geometry above the Griffith threshold.
\end{abstract}

The nonequilibrium physics of dynamic fracture continues to be a challenging topic of great interest [1]. Recent efforts have been spurred by experimental findings regarding the breakdown of straight crack propagation (along with its associated smooth fracture surface) as the crack speed exceeds a threshold value. This instability has been seen in a variety of materials, both crystalline [2] and amorphous [3,4], and it has been reproduced in molecular dynamics simulations [5] as well as with finite element schemes [6].

For any material undergoing brittle fracture, linear continuum elasticity only provides an accurate description of the displacements in regions that are not too close to the crack tip. The classic approach to this problem [1] has been to solve linear elastic equations, with boundary conditions providing the driving stresses, right up to this tip. This approach relies upon the assumption that the "process zone" inside of which the linear continuum equations break down is microscopic in size. The solutions have stress fields which become singular at the assumed tips, representing a finite flow of energy into the infinitesimally sized process zone. The velocity of the crack is then phenomenologically assumed to be given by some function of this energy flow rate.

This approach has two main limitations from a physics perspective. Firstly, it does not provide insight into how the crack velocity is actually determined, e.g. how it depends on short-scale dissipation. Secondly, and more importantly, it fails to predict instabilities of the tip dynamics. Thus, just as is the case in the well-studied problem of dendritic solidification [7], one must supplement the macroscopic transport physics with a consistent, regularizing microscopic theory on the tip scale in order to create a sensible theoretical framework.

One method for accomplishing this task involves placing the system on a lattice and allowing for the elastic forces to rapidly diminish at large atomic separation. Analytical [8, 11] and numerical [10,12] studies of such models have shown that the details of the lattice structure are critical for the tip dynamics. This is not surprising since in general the process zone scale is just the lattice spacing. Thus, these models are useful but can- not even qualitatively describe experiments in amorphous systems. What appears to be a more sensible approach for this class of systems is to construct a regularized continuum model that maintains rotational symmetry even inside the process zone. Constructing such a theory is the aim of this paper 13.

As a first step, we focus here on the simpler situation of mode III fracture for which the displacement $\vec{u}$ can be taken to be in a fixed direction (out of the plane) and hence can be represented by a scalar field $u$. Standard linear elasticity assigns the energy

$$
E=\int d \vec{x} \frac{1}{2} \mu \vec{\epsilon}^{2}
$$

with the strain $\vec{\epsilon}=\vec{\nabla} u$ and an elastic constant $\mu$. Allowing a material to fracture means that at large enough $\vec{\epsilon}^{2}$, the energy becomes strain independent, thereby eliminating the force. In an ideally brittle material, for example, this transition occurs immediately at some critical magnitude of the strain, $\epsilon_{c}$. Our basic idea involves representing the local state of the system, either unbroken with $|\epsilon|<\epsilon_{c}$ or broken with $|\epsilon|>\epsilon_{c}$, via a second "phase" field $\phi(\vec{x}, t)$. This field can be made to track the correct state if it obeys a standard two-minimum GinzburgLandau equation with the relative energy of the two wells dependent on $\epsilon^{2}-\epsilon_{c}^{2}$. Specifically, we choose

$$
\tau \partial_{t} \phi(\vec{x}, t)=D_{\phi} \nabla^{2} \phi-V_{D W}^{\prime}(\phi)-\frac{\mu}{2} g^{\prime}(\phi)\left(\vec{\epsilon}^{2}-\epsilon_{c}^{2}\right)
$$

where $V_{D W}(\phi)=\frac{1}{4} \phi^{2}(1-\phi)^{2}$ and $g$ is a function specified later that has the properties $g(0)=0, g(1)=1$ and $g^{\prime}(0)=g^{\prime}(1)=0$. With these choices, the two minima are always at $\phi=0$ and $\phi=1$, with the absolute minimum shifting from 1 to 0 as $\vec{\epsilon}^{2}$ passes $\epsilon_{c}^{2}$.

To close the system, we need to specify how $\phi$ affects the elasticity equation. Note that the above equation follows from the relaxational dynamics, $\tau \partial_{t} \phi=-\delta E / \delta \phi$, where the energy $E$ is now given by

$$
\int d \vec{x}\left[\frac{1}{2} D_{\phi}(\vec{\nabla} \phi)^{2}+V_{D W}(\phi)+\frac{\mu}{2} g(\phi)\left(\vec{\epsilon}^{2}-\epsilon_{c}^{2}\right)\right]
$$


If we now interpret this $E$ as the full potential energy including the elastic contribution, we see that the aforementioned properties of $g(\phi)$ will in fact eliminate the elastic force for large strain without having any effect at small strain where $\phi \simeq 1$ and thus $g \simeq 1$. Consequently, our second equation is derived by varying this energy with respect to displacement, which yields

$$
\rho \frac{\partial^{2} u}{\partial t^{2}}+b \frac{\partial u}{\partial t}=\mu \vec{\nabla} \cdot\left(g(\phi) \vec{\nabla}\left(1+\eta \frac{\partial}{\partial t}\right) u\right),
$$

where we have allowed for both a Stokes drag term with coefficient $b$ and a Kelvin viscosity $\eta$. This equation completes our model specification.

Let us place this work in some perspective. Our approach is similar in philosophy to, but very different in detail from, the work of Aranson and co-workers 16] who also derive a continuum two-field model for fracture. Most crucially, the physical interpretation of the phase field and hence the way in which it enters dynamically are completely different. As a result the present model avoids certain unphysical features of their model, e.g. the logarithmic dependence of the crack opening on the system size. Within the traditional fracture community, several researchers [17] have studied the effects of "softening" the elastic energy at large strain and compensating for the resultant instability in the equations by adding higher derivative terms. This approach, however, turns out to be difficult to extend to construct a continuum model where the fracture energy is independent of the system size and, at the same time, the strain is fully relieved in the bulk solid after passage of the crack. Moreover, it leads to a single fourth-order elasticity equation that is extremely hard to treat numerically. Finally, it is worth recalling that the original idea [18] of representing different phases of a system via a field coupled to the macroscopic dynamics, and thereafter using derivative terms in the phase field to regularize the problem, arose in the context of nonequilibrium crystal growth where it has become the method of choice [19,20] for highly accurate computations of solidification microstructures.

To understand how our model accounts for fracture, we start with the (one dimensional) snap-back of a stretched elastic band of size $2 L$ after it breaks in the middle. Let us first consider the final time-independent cracked state; note that this is equivalently the asymptotic state for a 2$\mathrm{d}$ crack once the tip has passed. This state is determined by solving the above equations with all time derivatives set to zero, with the boundary conditions $u( \pm L)= \pm \Delta$, $\phi( \pm L)=1$. Note that $2 \Delta$ is the total integrated strain that is conserved by the dynamics. Moreover, both $\epsilon(y)$ and $\phi(y)$ are symmetrical about $y=0$; thus we only need to find a solution in the interval $0 \leq y \leq L$. The elasticity equation, Eq. 田, requires $\epsilon(y)=\partial_{y} u=\epsilon_{0} / g(\phi(y))$. Substituting this form into Eq. 2 yields

$$
0=D_{\phi} \phi^{\prime \prime}-V_{D W}^{\prime}(\phi)-\frac{\mu}{2} g^{\prime}(\phi)\left(\frac{\epsilon_{0}^{2}}{g^{2}(\phi)}-\epsilon_{c}^{2}\right)
$$

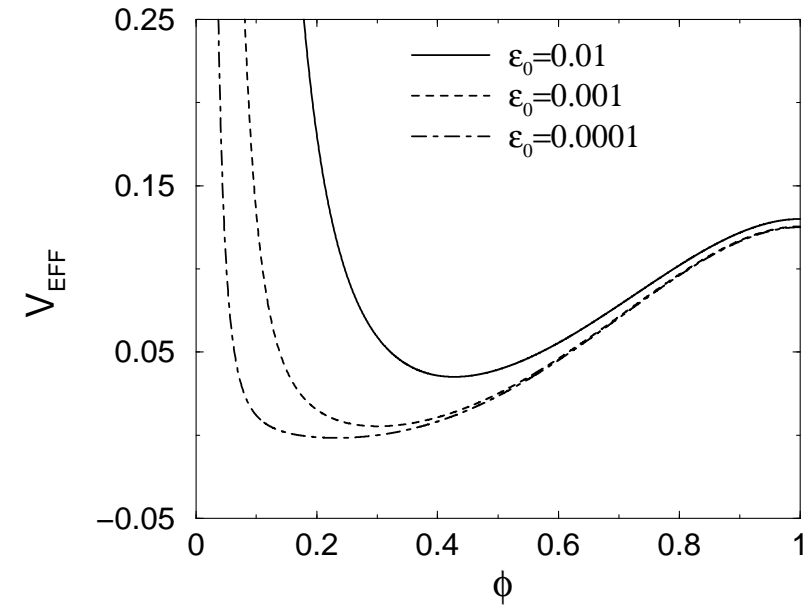

FIG. 1. Plots of the effective potential for 1-d static crack profiles $\left(\mu=1\right.$ and $\left.\epsilon_{c}=1 / 2\right)$.

From now on, we rescale lengths to make $D_{\phi}=1$. Eq. 5 can be thought of as the equation of motion of a ball rolling in an effective potential

$$
V_{E F F}(\phi)=-V_{D W}+\frac{\mu}{2}\left(g(\phi) \epsilon_{c}^{2}+\frac{\epsilon_{0}^{2}}{g(\phi)}\right)
$$

A schematic picture of this potential is shown in Fig. 1. The solution of interest corresponds to rolling in a "time" $L$ from the top of the hill at $\phi=1$ to the turning point $\phi^{*}$ located near $\phi=0$; this turning point exists because $\epsilon_{0}^{2} / g(\phi)$ becomes large and positive for small $\phi$.

The asymptotic steady-state crack is thus given by

$$
\frac{1}{\sqrt{2}} \int_{\phi^{*}}^{\phi(y)} \frac{d \tilde{\phi}}{\sqrt{E_{0}-V_{E F F}(\tilde{\phi})}}=y
$$

The two unknown constants $\epsilon_{0}$ and $E_{0}$ are fixed by the requirements that the above equation yields $\phi=1$ at $y=L$ and by the overall integrated strain constraint

$$
\frac{\epsilon_{0}}{\sqrt{2}} \int_{\phi^{*}}^{1} \frac{d \tilde{\phi}}{g(\tilde{\phi}) \sqrt{E_{0}-V_{E F F}(\tilde{\phi})}}=\Delta
$$

For this solution to be physically acceptable, almost all of the displacement must occur in the crack, thereby relieving the strain in the bulk, which imposes a constraint on the form of the function $g$. To see why, consider the large $L$ limit (where with $D_{\phi}=1$ our length unit is the process zone scale) and note that the standard Griffith criterion for fracture necessitates the scaling $\Delta \sim \sqrt{L}$. There is a contribution to the integral on the left hand side of Eq. 8 that arises from $\phi$ of order $\phi^{*}$, which is close to zero. If we choose $g$ to vanish near $\phi=0$ as a power law $g \sim \phi^{2+\alpha}$, it is clear from the form of the effective potential that $\phi^{*} \sim \epsilon_{0}^{\frac{2}{2+\alpha}}$. Given this, the local contribution to the integral scales like $\epsilon_{0}^{-\frac{\alpha}{2+\alpha}}$. This will match the right hand side with the choice 

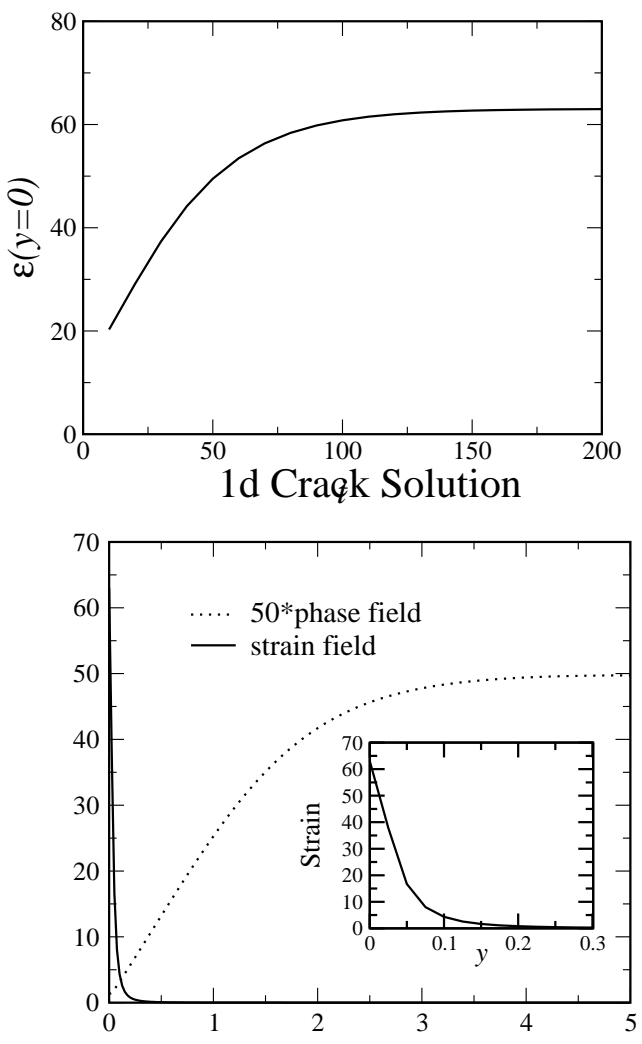

FIG. 2. Results of $1 \mathrm{~d}$ crack computations. (a) The strain at the crack center vs. time (b) asymptotic profiles of the strain and phase fields. $L=5, \epsilon_{0}=0.045$

$$
\epsilon_{0} \sim L^{-\frac{2+\alpha}{2 \alpha}}
$$

Hence, as long as $\alpha$ is positive, $\epsilon_{0}$ will go to zero at large $L$ fast enough such that the local contribution, i.e. that of the crack, to the overall displacement is dominant compared to the bulk contribution which scales as $\epsilon_{0} L$. We note that the least residual stress occurs in the limit $\alpha \rightarrow 0^{+}$, which gives rise to an exponential decrease as a function of system size. Finally, the fracture energy $\gamma$ remains finite as $L$ gets large, as is also required for a sensible theory. In this limit, $\epsilon_{0} \rightarrow 0$ and $E_{0} \rightarrow \mu \epsilon_{c}^{2} / 2$, and it is easy to derive the expression

$$
\gamma=\sqrt{2} \int_{0}^{1} d \tilde{\phi} \sqrt{\mu \epsilon_{c}^{2} / 2-V_{E F F}\left(\tilde{\phi} ; \epsilon_{0}=0\right)}
$$

We now turn to the $1 \mathrm{~d}$ time-dependent problem. We choose $g=4 \phi^{3}-3 \phi^{4}$, so that $\alpha=1$ and $\epsilon_{0}$ should scale as $L^{-3 / 2}$; also we pick $\epsilon_{c}=1 / 2, \mu=1$. Fig. 2 shows the time development of the strain at the origin, for a completely overdamped system, $\rho=0, b=1, \eta=0$. We see that, starting from an initial large strain region near the origin, the system proceeds to fully crack and approach the aforementioned asymptotic state. Notice that for this case of overdamped dynamics, there are two time scales visible in the strain relaxation; a fast time scale during which the crack (say in the $\phi$ field) develops and a slower one during which strain is drained from the bulk into the crack. For a typical underdamped case, ( $\rho=1, b=0, \eta=0.2$ ), the second-stage relaxation to the steady-value involves, as it must, damped oscillations. In either case, in the second stage, the $\phi$ field is slaved to the displacement making the system of equations very stiff. We coped with this difficulty using an implicit timestepping scheme.

The real test of any fracture model comes in two (or higher) dimensions. Now, the crack tip must advance by providing enough stress to strain new material beyond a critical extension. We have carried out a preliminary simulation study of our model in a 2-d strip geometry (with the edges of the strip at $y= \pm L$ ) using a standard Crank-Nicholson alterning-direction-implicit scheme 21. We used the initial condition corresponding to a strained solid with $\phi(x, y)=1$ and $u(x, y)=\Delta y / L$, which must produce a crack propagating along the $x$ direction above the standard Griffith threshold, $\Delta>\Delta_{c}$, where here $\Delta_{c}=\sqrt{2 \gamma L}$ and $\gamma=0.3808 \ldots$ is given by Eq. 10 for the present model parameters $\left(\mu=1, \epsilon_{c}=1 / 2\right)$. A typical time sequence for a stably propagating crack is presented in Fig. 3. We have checked that our results are reasonably independent of the discretization scale $d x$, such that we are truly seeing the results of the continuum regularization of the tip scale dynamics.

In Fig. 4, we present the steady-state crack velocity as a function of the driving. The crack propagates above a critical value of the drive that is within a few percent of the analytically predicted value $\Delta_{c}=\sqrt{2 \gamma L}$ for the large $L$ limit even though $L$ is not so large $(L=10)$ in the simulations. It is important to recognize that $V(\Delta)$ cannot be obtained from the usual continuum theory without additional assumptions; here it follows directly from the fact that we have a consistent theory at both the macroscopic and microscopic scales. One can obtain similar results from lattice models of fracture [9, 10,23, at the price of introducing lattice scale instabilities [22,12]. These instabilities are connected to spatial period-doubling, when the times for breaking alternating diagonal bonds in a hexagonal lattice become unequal. Thus, they have no direct relevance for amorphous systems without any underlying crystallinity. Here, at least at the drivings we have investigated so far, we find no such instability, and steady cracking appears to persist to rather high displacements. In terms of experiment, this seems to imply that the observed transition at moderate displacement to more complex tip dynamics is presumably connected to the mode I nature of the fracture geometry, with its propensity to branch in the direction of the off-axis stress maximum as suggested originally by Yoffe 24]. An extension of the present approach to mode I is presently underway to test this hypothesis.

In terms of physics, our approach leads to the introduction of a new time scale, $\tau$, connected to the relaxational rate of the phase-field. At any non-zero $\tau$, this relaxation is a possible source of tip-scale dissipation and hence it can affect the crack propagation. Decreasing this pa- 


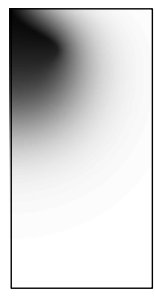

(a)

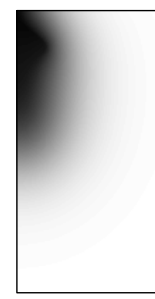

(b)

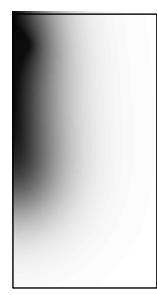

(c)

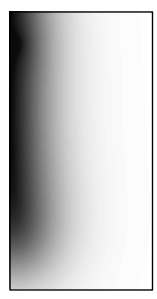

(d)
FIG. 3. 2-d simulation snapshots; pictured here is $\phi$ in grey-scale $(0=$ black, $1=$ white) over $1 / 2$ the computational domain of size 20x20; here $\rho=1, b=0, \eta=.2, \Delta=2.81$, timestep $=.02$ and grid spacing $=.05$

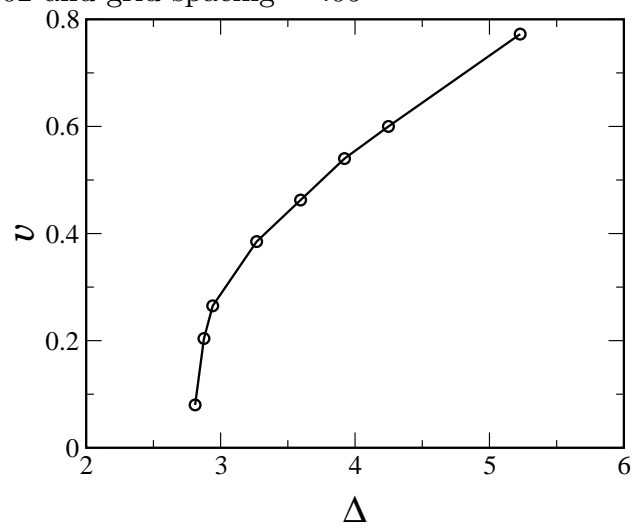

FIG. 4. Velocity vs. driving from 2-d simulations.

rameter in the simulations indeed increases the velocity, consistent with convergence to a finite limit as $\tau \rightarrow 0$ with $O(\tau)$ corrections. This indicates that the velocity is predominantly limited here by the rate at which the strain is drained from the bulk into the crack.

An important numerical issue for future consideration concerns the sharpness of the strain profile. To fully resolve the spatial scales in the 2-d crack is a daunting task which probably cannot be accomplished by sticking with a fixed computational grid. A related issue concerns the long time scale necessary for full strain relaxation. It may be possible to modify the time derivative terms in the equation of motion to speed up this relaxation. Furthermore, to make contact with expected results from the fracture community (such as the role of the stress intensity factor and the fact that crack tips will propagate without inertia at least until times such that shear wave reflections from the boundaries can affect the motion) will require much larger systems and much more attention to details of the initial conditions.

The authors thank the hospitality of the Aspen Center for Physics, where this work was begun. DAK acknowledges the support of the Israel Science Foundation. AK acknowledges support of DOE and thanks Jim Rice for valuable discussions in the initial stage of this work.
[1] J. Fineberg and M. Marder, Phys. Repts. 313, 1-108 (1999); K. Broberg, ",Cracks and Fractures", Academic Press (1999); L. B. Freund, "Dynamic fracture mechanics", Cambridge University Press, 1990.

[2] T. Cramer, A. Wanner, and P. Gumbsch, Z. Metallkd. 90, 675-686 (1999); Phys. Rev. Lett. 85, 788-791 (2000).

[3] J. Fineberg, S. P. Gross, M. Marder, and H. L. Swinney, Phys. Rev. Lett. 67, 457-460 (1991); Phys. Rev. B 45, 5146-5154 (1992); K. Ravi-Chandar and W. G. Knauss, Int. J. Fracture, 25, 247 (1984);

[4] E. Sharon, et al, Phys. Rev. Lett. 74, 5096-5099 (1996); Phys. Rev. Lett. 76, 2117 (1996).

[5] F. F. Abraham, et. al., Phys. Rev. Lett. 73 (2), 272-275 (1994); S. J. Zhou, D. M. Beazley, P.S. Lomdahl, and B. L. Holian, Phys. Rev. Lett. 78, 479 (1997) ; P. Gumbsch, S. J. Zhou, and B. L. Holian, Phys. Rev. B 55, 3445 (1997); D. Holland and M. Marder, Phys. Rev. Lett. 80, 746 (1997); A. Omeltchenko, J. Yu, R. K. Kalia, and P. Vashishta, P., Phys. Rev. Lett. , 78, 2148-2151 (1997).

[6] X.-P. Xu and A. Needleman, J. Mech. Phys. Solids 42, 1397 (1994); E. Johnson, Int. J. Fract., 57, R27 (1992).

[7] D. A. Kessler, J. Koplik and H. Levine, Adv. Phys. 37, 255 (1988).

[8] L. I. Slepyan, Doklady Akademii Nauk SSSR 258, 561 (1981) Sov. Phys. Dokl. 26, 538 (1981)); Sh. A. Kulamekhtova, V. A. Saraikin and L. I. Slepyan, Mech. Solids 19, 102 (1984).

[9] M. Marder and S. Gross, J. Mech. Phys. Sol. 43, 1 (1995).

[10] D. A. Kessler and H. Levine, Phys. Rev. E 59, 5154 (1998); D. A. Kessler, Phys. Rev. E 61, 2348 (2000).

[11] L. Pechenik, H. Levine and D. A. Kessler, preprint.

[12] D. A. Kessler and H. Levine, Phys. Rev. E in press.

[13] Attempts to do this by merely modifying the boundary conditions (so-called cohesive zone models [14]) have not been successful to date [15].

[14] G. I. Barenblatt, Appl. Math. and Mech. 23, 622-636 (1959); J. S. Langer, Phys. Rev. A 46, 3123 (1992).

[15] J.S. Langer, and A. E. Lobkovsky, J. Mech. Phys. Sol. 46, 1521 (1998).

[16] I. S. Aranson, V. A. Kalatsky and V. M. Vinokur, Phys. Rev. Lett. 85, 118-21 (2000).

[17] Z. Pazant, App. Mech. Rev. 39, 675 (1986). This work was mostly focused on shear banding, but can be generalized to fracture.

[18] J. B. Collins and H. Levine, Phys. Rev. B 31, 6118-22 (1985); J. S. Langer, in Directions in Condensed Matter Physics, edited by G. Grinstein and G. Mazenko (World Scientific, Philadelphia, 1986; G. Caginalp and P. Fife, Phys. Rev. B 33, 7792-4 (1986).

[19] A. Karma and W. Rappel, Phys. Rev. E 57, 4323 (1998).

[20] N. Provatas, et. al, Phys. Rev. Lett. 82, 4496-9 (1999).

[21] William H. Press, et al, "Numerical recipes in FORTRAN : the art of scientific computing" 2nd ed., Cambridge University Press, 1992.

[22] M. Marder and X. Liu, Phys. Rev. Lett. 71, 2417 (1993).

[23] L. I. Slepyan, M. V. Ayzenburg-Stepanenko, and J. P. Dempsey, Mechanics of Time-Dependent Materials 3, 159-203 (1999).

[24] E. Y. Yoffe, Philos. Mag. 42, 739-750 (1951). 